\title{
Achieving coronal plane alignment in total knee arthroplasty through modified preoperative planning based on long-leg radiographs: a prospective study
}

\author{
Daria Singh ${ }^{1^{*}}$, Kalpeshkumar C. Patel ${ }^{1}$ and Ragini D. Singh ${ }^{1,2}$
}

\begin{abstract}
Level of evidence: ॥

\section{Background}

Total knee arthroplasty (TKA) is a reliable surgical intervention to treat pain and disability caused by advanced arthritis of the knee. The utilization of TKA is increasing at a fast pace worldwide, owing to the increasing aging population and improved outcomes of TKA. The demand for TKA is also increasing in young, active patients with knee arthritis who expect a better functional outcome and longer implant
\end{abstract}

Purpose: This prospective study was undertaken to examine whether the desired coronal plane alignment of limb and prosthetic components in total knee arthroplasty (TKA) could be achieved precisely using conventional jig-based methods by modifying the preoperative planning of bone resection utilizing long-leg radiographs (LLRs).

Methods: The study included consecutive 245 TKA procedures. Pre- and postoperative radiological variables, i.e., the mechanical axis (hip-knee-ankle [HKA] axis), mechanical lateral distal femoral angle (mLDFA), and medial proximal tibial angle (MPTA), and their outliers were evaluated. Statistical analysis was performed using SPSS version 21.0.

Results: The mean postoperative HKA axis, mLDFA and MPTA was $179.80 \pm 1.81^{\circ}(p<0.01$; 95\% Cl: 8.09-9.67), $90.35 \pm 1.54^{\circ}(p<0.01 ; 95 \% \mathrm{Cl}: 1.33-2.02)$, and $90.26 \pm 1.25^{\circ}(p<0.01 ; 95 \% \mathrm{Cl}: 4.41-5.20)$, respectively. The postoperative HKA axis on the coronal plane was $180 \pm 3^{\circ}$ in 235 knees (95.92\%, 4.08\% outliers). Femoral and tibial components were implanted in an acceptable position, withing $90 \pm 3^{\circ}$ of the mechanical axis of the femur and tibia on the coronal plane in 238 (97.14\%, 2.86\% outliers) and 243 (99.18\%, 0.8\% outliers) knees, respectively.

Conclusion: Modified preoperative planning for TKA on LLRs is a reliable and consistent method to achieve the desired limb and component alignment on the coronal plane without adding financial or logistical costs.

Keywords: Conventional TKA, Preoperative planning, True-alignment technique

*Correspondence: drdariasingh@yahoo.co.in

1 Zydus Hospitals, Ahmedabad, Gujarat 380059, India

Full list of author information is available at the end of the article survival [35]. Due to the large absolute number of TKA procedures being performed, even a small percentage of error can lead to a very large absolute number of TKA procedures being at risk for poor functional outcomes, patient dissatisfaction, and early implant failure. Neutral mechanical alignment $\left(180 \pm 3^{\circ}\right)$ on the coronal plane is one of the most important determinants of successful TKA [1]. Many published studies have suggested that TKA with good alignment on the coronal plane leads to a better functional outcome and enhanced patient satisfaction [10, 16, 23]. The neutral mechanical axis reduces contact stresses on the implant and its subsequent wear [22, 39] and related failures, 
and stresses on the bone-implant interface and thereby enhances implant survival [14, 40]. A frequently cited study by Parratte et al. observed that better alignment did not lead to increased implant survival. However, the authors also concluded that until additional data can be generated to determine the ideal postoperative alignment in an individual patient, the neutral mechanical axis remains a reasonable target [30].

There are various alignment options, including anatomical, anatomical-like, mechanical, adjusted mechanical, kinematic, and restricted kinematic alignment, to recreate a mechanical environment conducive to a better functional outcome and increased implant survival [33]. However, an overwhelmingly large number of studies support the concept of mechanical alignment [1]. There are various surgical techniques, such as conventional surgery, optimized conventional surgery [26], computerassisted surgery (CAS) [4], patient-specific instrumentation (PSI) [6], and robot-assisted surgery [8], to achieve the target limb and component alignment according to the alignment option adopted. Despite technological advances and improvements in understanding, achieving neutral mechanical alignment on the coronal plane remains a daunting task. In conventional jig-based methods, the rate of achieving neutral alignment is abysmally low because of large anatomical variabilities [34, 38], a fixed valgus cutting angle (VCA) [12, 27], aberrant jig placement [28], intraoperative cutting errors [7], the lack of a consensus on the proximal tibial landmark for fixing tibial jigs $[2,11,15]$ and the inherent limitations of jigs [9, 32]. Various technologies, such as CAS, PSI, and robot-assisted TKA, have been developed in an effort to improve the rate of achieving neutral mechanical alignment, but all of these methods included additional variable costs and the potential for complications unique to the technology used [37]. These technologies are increasingly being used in the developed world. However, the affordability and availability of these technologies are still problems in developing countries, where increasingly large numbers of TKA procedures are being done with conventional methods [3, 31].

Therefore, the present study aimed to determine whether a modified preoperative planning method (i.e., the true-alignment technique) based on long-leg radiographs (LLRs) can consistently achieve neutral mechanical alignment of the leg as well as alignment of the femoral and tibial components in TKA using conventional jigs while not incurring any additional cost or increasing the risk of complications. Given current knowledge, the hypothesis was that a neutral mechanical limb axis and femoral and tibial component alignment on the coronal plane could be consistently achieved with the method described in the present paper.

\section{Material and methods}

\section{Study design}

This noncomparative, open-label, single-arm clinical study was carried out after ethics committee approval was obtained. The sample size was calculated using an equality test for a one-sample design (power of $80 \%, \alpha$ of 0.05 and standard deviation of 1.7 for the mechanical axis) [26]. Based on these estimates, a total of 235 cases were required. Considering a 5\% dropout and/or withdrawal rate, $N=245$ knees were included. The inclusion criteria comprised informed consent, coronal plane deformity $<25^{\circ}$ varus or valgus, sagittal plane deformity $<15^{\circ}$, and satisfactory LLRs. Knees with $>25^{\circ}$ coronal plane deformity, $>15^{\circ}$ sagittal plane deformity, or unsatisfactory LLRs were excluded. We screened 239 consecutive patients who underwent TKA between October 2018 and March 2019 and included 191 patients (245 knees) and excluded 48 patients (48 knees). Twenty-three knees with coronal plane deformity $>25^{\circ}$, 13 knees with sagittal plane deformity $>15^{\circ}$ and 8 knees with poor-quality LLRs (nonvisualization of the center of the femoral head) were among the excluded knees. Four knees were excluded due to nonavailability of LLRs due to X-ray machine breakdown. No patients were excluded or dropped out postoperatively.

\section{Surgical planning}

All surgeries were planned and performed by the first author (DS). As a reliable tool for measuring the mechanical axis and component position, standing LLRs were used to measure all pre- and postoperative radiological parameters and plan proximal tibial and distal femoral bone resection [13]. Standardized digital LLRs were obtained in the standing position by a previously described method [13]. Radiographs were evaluated for acceptable image quality and rotation by assessing the profile of the lesser trochanter, the positioning of the patella over the femur (central position was accepted), and the amount of overlap of the fibular head on the tibia (one-third of fibular head overlap was accepted). The radiographs were considered satisfactory when the above criteria for at least two landmarks were met. Once satisfactory radiographs were obtained, the patients were included in the study. Postoperative standing LLRs were obtained when patients were able to complete a full active straight-leg raise and stand straight on the radiography platform. The following pre- and postoperative coronal plane radiological parameters were measured on a digital radiograph workstation: (i) the mechanical axis (hip-knee-ankle [HKA] axis), i.e., the angle between the femoral mechanical axis (line joining the center of the femoral head and the center of the femoral/femoral implant trochlear notch), and the tibial mechanical axis, 


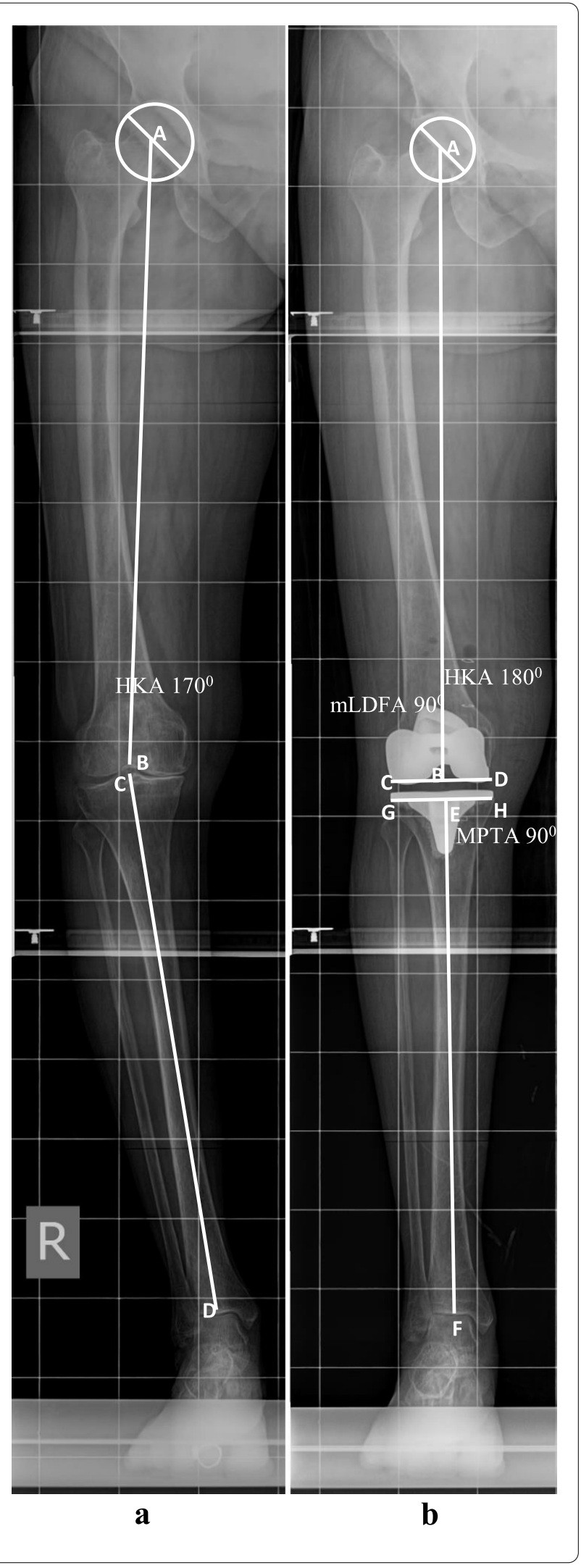

Fig. 1 a Preoperative standing LLR denoting the centre of femoral head (point A), centre of the distal femoral articular surface (point B), femoral mechanical axis (Line AB), centre of the proximal tibia/ centre of tibial spine (point $C$ ), centre of the distal tibial articular surface, coinciding with midtalar groove (point D), HKA angle i.e. angle between line $A B$ and $C D$ is $168^{\circ}$ or $12^{\circ}$ varus. $\mathbf{b}$ Postoperative standing LLR denoting HKA angle, (angle between line $A B$ and $E F$ ) $180^{\circ}, \mathrm{mLDFA}$, angle $\mathrm{ABC}, 90^{\circ}$ and MPTA, angle FEH, $90^{\circ}$

i.e., the line joining the point between the tibial spine/ center point of the tibial base plate to the central depression on the dome of the talus; (ii) the mechanical lateral distal femoral angle (mLDFA), i.e., the lateral angle between the femoral mechanical axis and the tangent line connecting the distal femoral condyle preoperative and femoral prosthesis postoperative; and (iii) the medial proximal tibial angle (MPTA), i.e., the medial angle between the tibial mechanical axis and the tangent line connecting the proximal tibial condyles preoperative and tibial component postoperative. The aim was to restore the mechanical axis classically within $3^{\circ}$ of the neutral mechanical axis (HKA axis $180 \pm 3^{\circ}$ ).

The second author (KCP) measured and recorded all the pre- and postoperative outcome variables. Since he was not the operating surgeon, the use of this method also helped to avoid any operator bias. The outcome variables of 20 randomly selected knees were remeasured after 2 weeks by KCP. These variables in the same set of knees were also measured by DS to test the intraobserver and interobserver variability.

\section{Distal femoral resection planning}

The aim of distal femoral resection was to cut the distal femur at $90^{\circ}$ to its mechanical axis. The femoral mechanical axis (line $A B$ ) joining the center of the femoral head (point A) and the deepest point of the intercondylar notch (point B) on LLRs (Figs. 1a \& 2a) was drawn. Then, a second line (CD) was drawn tangent to the distal femoral articular surface. This angle $A B C$ was the mLDFA (Fig. 2a). If the mLDFA was $90^{\circ}$, then bone cuts of equal thickness for both distal femoral condyles were planned. If the mLDFA was $>90^{\circ}$ or $<90^{\circ}$, then another line $(\mathrm{CD})$ perpendicular to the mechanical axis of the femur (line $\mathrm{AB}$ ) and tangent to at least one femoral condyle was drawn. The length of line EF was the difference in bone resection between the two condyles (Fig. 2b). Since intramedullary jigs were used to guide resection, the VCA were calculated by drawing a line from the deepest point of the trochlear groove (portal of entry) to the center of the medullary canal at $23 \mathrm{~cm}$ from the portal of entry (line BG). Line BG was the trajectory for the intramedullary rod, and angle $\mathrm{GBC}$ was the 


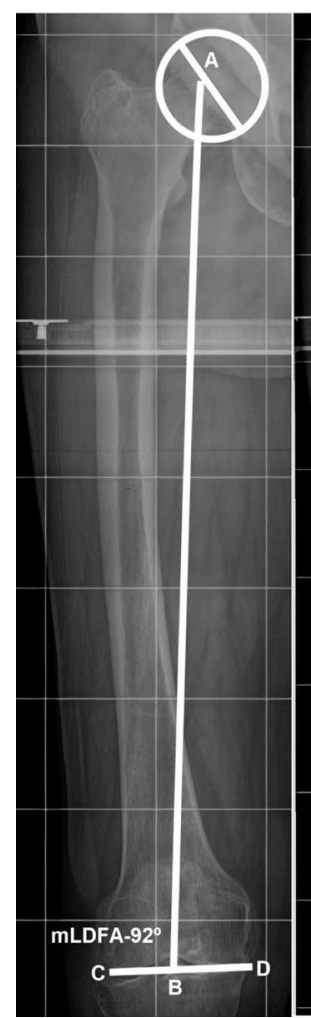

a

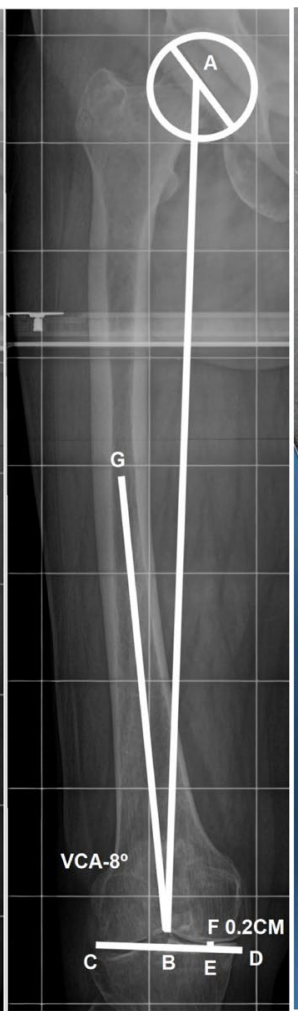

b

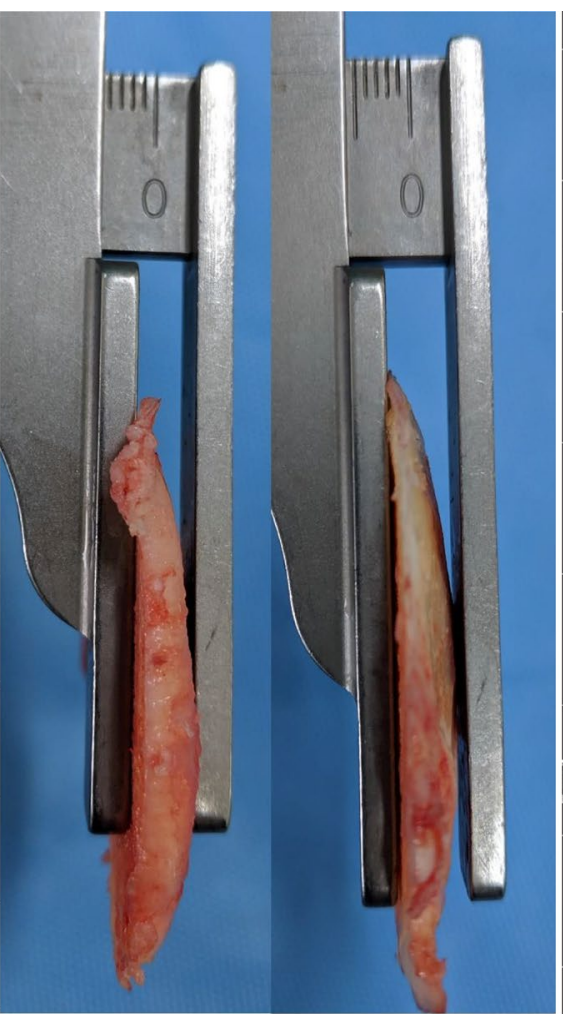

d

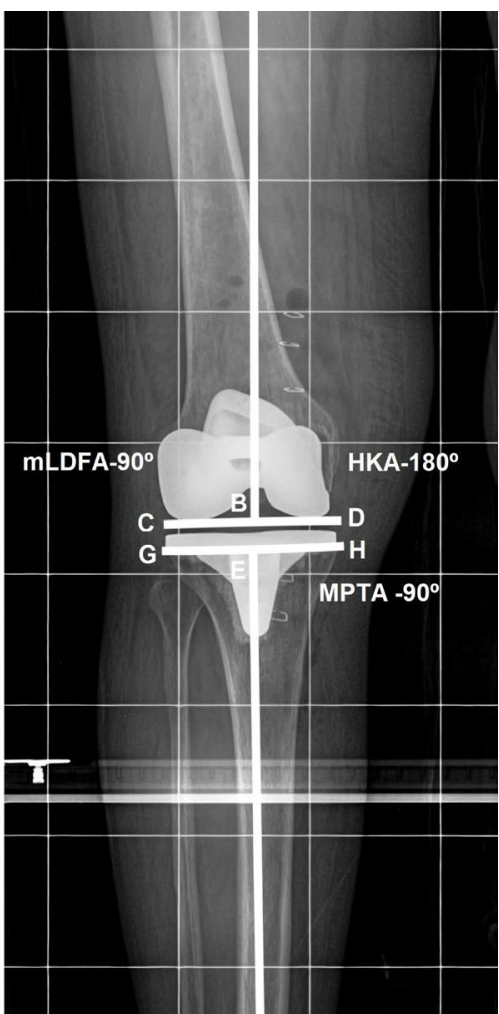

e

Fig. 2 a Depicting the mechanical axis of femur (Line AB), and a tangential line to the distal femoral articular surface (Line CD) and preoperative mLDFA (angle $A B C$ ), $92^{\circ}$ that means $2^{\circ}$ varus correction in femur is required. $\mathbf{b}$ Depicting the femoral mechanical axis (Line AB), and a line perpendicular to mechanical axis, a line tangential to at least one distal femoral condyle, and in this case lateral distal femoral condyle, (Line CD). Line CD is $0.2 \mathrm{cms}$ away from medial femoral condyle, meaning bone cut from medial femoral condyle must be $0.2 \mathrm{~cm}$ less than lateral femoral condyle. Line GB is trajectory for intramedullary road and this line makes $82^{\circ}$ angle (angle GBC), which means VCA of $8^{\circ}$. c Intra-operative photograph showing thickness of the bone resected from the medial femoral condyle, $4.5 \mathrm{~mm}$. $\mathbf{d}$ Intra-operative photograph showing the thickness of the bone resected from the lateral femoral condyle, $6 \mathrm{~mm}$ (planned difference of thickness of bone resection from medial and lateral femoral condyle was $2 \mathrm{~mm}$, here we could get $1.5 \mathrm{~mm}$ difference). e Post-operative LLR (showing only knee). Depicting the $\mathrm{mLDFA}$, angle $A B C, 90^{\circ}$ (planned femoral component position)

planned VCA (pVCA) (Fig. 2b). Intraoperatively, bone to be resected from the distal femoral condyle with the pVCA was measured after removing cartilage from the unaffected condyle. If it matched the preoperative plan for bone resection, then the same bone thickness was resected. If it did not match, then the VCA was adjusted to the planned bone resection thickness. The final VCA read on the jig was the executed VCA (eVCA). The femoral component position was confirmed on postoperative LLRs (Fig. 2c, d \& e).

\section{Proximal tibial resection planning}

Proximal tibial resection was planned at $90^{\circ}$ to the tibial mechanical axis. The tibial mechanical axis (line $A B$ ) was drawn using the center of the tibial spine (point A) and the deepest point of the superior articular surface of the talus (point B) (Fig. 3a). The second line CD was drawn tangent to both proximal tibial condyles. The angle $\mathrm{BAD}$ was the MPTA. Then, a line (CD) tangent to the unaffected proximal condyle and perpendicular to the tibial mechanical axis (AB) was drawn, and the difference in the thickness of the bone to be resected was measured, i.e., the length of line EF (Fig. 3b). Intraoperatively, the mark on the superior surface of the cutting block of the extramedullary tibial jig was aligned to a line drawn on the center of the tibial spine from anterior to posterior ignoring the position of the tibial tubercle. On the unaffected side, the planned depth of resection required for the thinnest tibial component was measured, with a minimum of $9 \mathrm{~mm}$ for the ATTUNE Knee System (Johnson and Johnson), including bone and cartilage. Then, cartilage from the unaffected proximal tibial condyle was removed, and the thickness of bone to be resected $(9 \mathrm{~mm}$ minus the cartilage thickness) was remeasured and matched to the preoperative plan. Similarly, the thickness of bone to be resected on the affected side was measured 


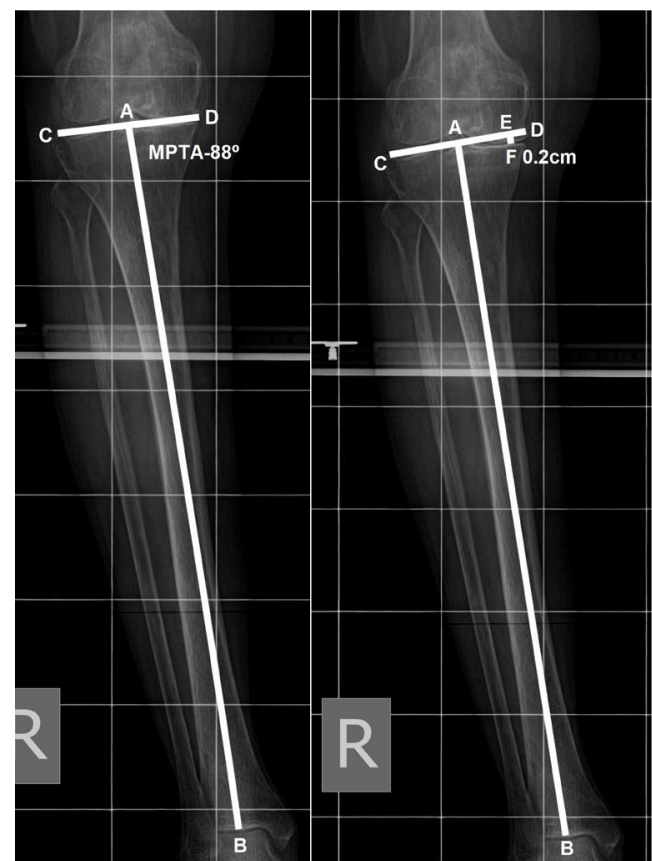

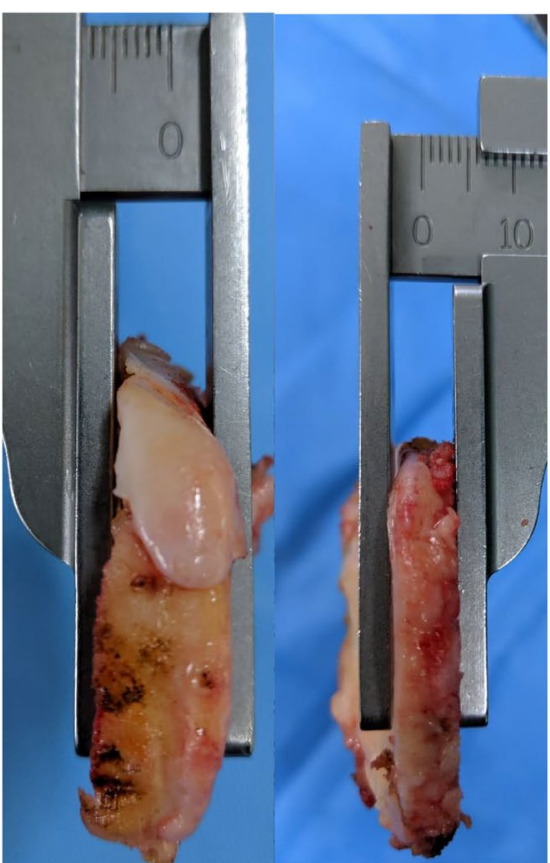

c

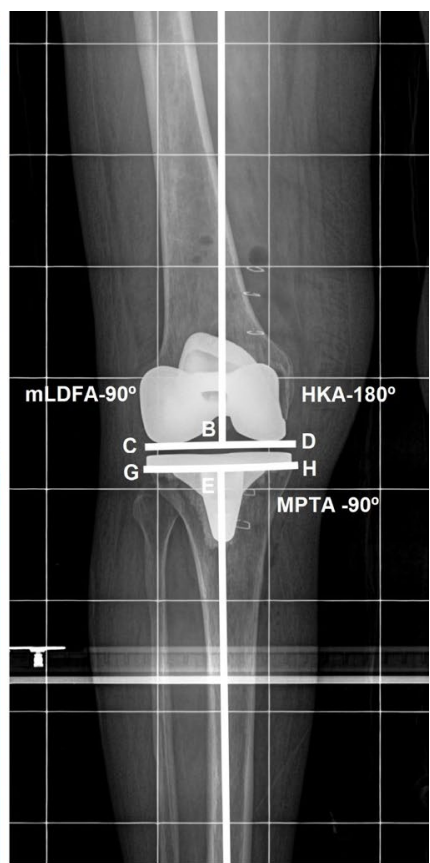

e

Fig. 3 a LLR (showing only tibia). Point A - a point in the middle of both tibial spine, point B coinciding with depression on dome of talus, line $A B$ - tibial mechanical axis, line CD - A tangential line to the both proximal tibial condyles, Angle ABD is MPTA $\left(88^{\circ}\right)$, meaning proximal tibia $2^{\circ}$ varus. $\mathbf{b}$ Drawing of planned proximal tibial resection, line $A B$ mechanical axis, line $C D$ is perpendicular to the mechanical axis passing through point $A$ and tangential to unaffected proximal tibial condyle, here in this case lateral tibial condyle. Line EF perpendicular to Line CD and point $F$ is deepest point at margin of medial tibial condyle, length of this line denotes difference between planned thickness of proximal tibial resection, here planned tibial resection was $2 \mathrm{~mm}$ more from lateral tibial condyle than medial tibial condyle after removing cartilage from lateral tibial condyle (with cartilage, depth of resection was planned $9 \mathrm{~mm}$ from unaffected condyle, lateral condyle in this case). $\mathbf{c}$ Intra-operative measurement of thickness of the resected proximal lateral tibia, $8 \mathrm{~mm}$ ( $2 \mathrm{~mm}$ more than medial proximal tibial condyle). $\mathbf{d}$ Intra-operative measurement of thickness of the resected proximal medial tibia, $6 \mathrm{~mm}$. e Postoperative LLR (showing only knee) illustrating MPTA, angle FEH, $90^{\circ}$, planned tibial component position

and matched to that in the preoperative plan. If there was any discrepancy, then the jig was adjusted accordingly. The thickness of the resected bone was measured and matched to that in the preoperative plan and confirmed on postoperative LLRs (Fig. 3c, d \& e).

The procedures were performed under combined spinal-epidural or spinal anesthesia. General anesthesia was given when spinal and epidural anesthesia were contraindicated. All procedures were performed using a tourniquet except in patients with heavily calcified arteries in the lower limbs. The ATTUNE Knee System (J\&J) was implanted using parapatellar arthrotomy. Routine perioperative antibiotics and thromboprophylaxis (enoxaparin) were administered for $48 \mathrm{~h}$ and 5 days, respectively.

\section{Outcome measures}

All demographic data and outcome variables were measured and recorded prospectively by KCP. The radiological variables (outcome variables) recorded were the pre- and postoperative mechanical axis (HKA axis),
mLDFA, and MPTA. Other radiographic measures recorded were the pVCA and eVCA.

\section{Statistical analysis}

The data were analyzed using SPSS statistical software (version 21.0, IBM Corp., Armonk, N.Y., USA). Determination of the change in the outcome parameters between preoperatively and postoperatively was carried out using a paired -sample t-test. Correlations between the HKA axis, mLDFA, and MPTA were assessed using Pearson's correlation coefficient. The intraobserver and interobserver variability were also evaluated. Fischer's exact test was applied to compare the percentage of outliers in the radiological parameters between groups stratified by age, sex, side, body mass index (BMI), and type and severity of deformity. $P$ values $\leq 0.05$ were considered to indicate statistical significance. 
Table 1 Demographic details of the study cohort $(N=245)$

\begin{tabular}{|c|c|}
\hline Age (years) & $\begin{array}{l}\text { Mean } \pm S D=63.93 \pm 7.29 \\
\text { Range: } 46.0-84.0 \\
95 \% \text { Cl: } 62.89-64.99\end{array}$ \\
\hline \multicolumn{2}{|l|}{ Sex } \\
\hline Male & $44(23.04 \%)$ \\
\hline Female & $147(76.96 \%)$ \\
\hline BMI $\left(\mathrm{kg} / \mathrm{m}^{2}\right)$ & $\begin{array}{l}\text { Mean } \pm S D=30.27 \pm 4.81 \\
\text { Range: } 22.0-49.5 \\
95 \% \text { Cl: } 29.27-30.76\end{array}$ \\
\hline \multicolumn{2}{|l|}{ Side } \\
\hline Left & $117(47.75 \%)$ \\
\hline Right & $128(52.25 \%)$ \\
\hline Unilateral & 135 knees (55.1\%) \\
\hline Bilateral & 110 knees (44.9\%) in 56 patients $^{\mathrm{a}}$ \\
\hline \multicolumn{2}{|l|}{ Deformity } \\
\hline Varus & 222 (90.61\%) \\
\hline Valgus & $18(7.34 \%)$ \\
\hline Neutral & 05 (2.04\%) \\
\hline
\end{tabular}

a One limb each in two patients had coronal plane deformity more than $25^{\circ}$ leaving a total number of 110 knees, in the bilateral group $170.91^{\circ} \pm 6.56^{\circ}(p<0.01 ; 95 \%$ CI: 8.09-9.67) (Table 2). The HKA axis was within $180 \pm 3^{\circ}$ in 235 knees (95.92\%) and outside this range in 10 knees (outliers, 4.08\%). All the outliers were in the preoperative varus deformity group $(10 / 227,4.40 \%)$. These outliers were further stratified according to the severity of deformity, as follows: $\leq 15^{\circ}$ in $7 / 193(3.62 \%)$ and $>15^{\circ}$ in $3 / 34$ (8.82\%) (Table 3$)$.

\section{Femoral and tibial component coronal plane alignment}

Femoral components were implanted at a mean mLDFA of $90.35 \pm 1.54^{\circ}$. The mean preoperative mLDFA was $88.68 \pm 2.59^{\circ} \quad(p<0.001 ; 95 \%$ CI: $1.33-2.02)$ (Table 2). The mean pVCA was $5.92 \pm 1.08^{\circ}$ (range: $3-9^{\circ}$ ), and the mean eVCA was $5.91 \pm 1.15^{\circ}$ (range: $3-10^{\circ}$ ). A total of 238 femoral components $(97.14 \%)$ were implanted within $90 \pm 3^{\circ}$ of the mLDFA. The eVCA was different from the conventional range of $5-7^{\circ}$ in $32 / 245$ (13.06\%) knees and different from the pVCA in 20 (8.16\%) knees. Tibial components were implanted at a mean of $90.26 \pm 1.25^{\circ}$. The mean preoperative MPTA was $85.41 \pm 3.38^{\circ}(p<0.01$; 95\% CI: 4.41-5.20). A total of 243 tibial components

Table 2 Pre- and postoperative primary outcome variables (Radiographic results)

\begin{tabular}{|c|c|c|c|}
\hline Radiographic variable & $\begin{array}{l}\text { Preoperative } \\
\text { Mean } \pm \text { SD and Range } \\
95 \% \mathrm{Cl}\end{array}$ & $\begin{array}{l}\text { Postoperative } \\
\text { Mean } \pm \text { SD and Range } \\
95 \% \mathrm{Cl}\end{array}$ & $p$ value; $95 \% \mathrm{Cl}$ \\
\hline HKA axis $\left({ }^{0}\right)$ & $\begin{array}{l}170.91 \pm 6.56 \\
\text { (Range: } 156-199) \\
95 \% \text { Cl: } 170.08-171.74\end{array}$ & $\begin{array}{l}179.80 \pm 1.81 \\
\text { (Range: } 174-185) \\
95 \% \text { Cl: } 179.56-180.02\end{array}$ & $<0.01 ; 8.09-9.67$ \\
\hline $\operatorname{mLDFA}\left({ }^{0}\right)$ & $\begin{array}{l}88.68 \pm 2.59 \\
\text { (Range: } 81-96) \\
95 \% \text { Cl: } 88.35-89.00\end{array}$ & $\begin{array}{l}90.35 \pm 1.54 \\
\text { (Range: 86-95) } \\
95 \% \text { Cl: } 90.16-90.55\end{array}$ & $<0.01 ; 1.33-2.02$ \\
\hline $\operatorname{MPTA}\left({ }^{0}\right)$ & $\begin{array}{l}85.41 \pm 3.38 \\
\text { (Range:76-102) } \\
95 \% \text { Cl: } 84.97-85.82\end{array}$ & $\begin{array}{l}90.26 \pm 1.25 \\
\text { (Range: } 86-94) \\
95 \% \text { Cl: } 90.09-90.41\end{array}$ & $<0.01 ; 4.41-5.20$ \\
\hline
\end{tabular}

\section{Results}

There were 245 knees in 191 patients, including 147 (76.96\%) females and $44(23.03 \%)$ males, with a mean age of $63.93 \pm 7.29$ years. A total of 56 patients (112 knees) underwent TKA on both sides simultaneously; of these, two knees in two patients were excluded because the preoperative coronal plane deformity was more than $25^{\circ}$ (110 knees were included from the bilateral group), while 135 patients (135 knees) underwent TKA on one side. Other demographic details and patient characteristics are provided in Table 1.

\section{Outcome variables HKA axis}

Postoperatively, the HKA axis was restored to a mean of $179.80 \pm 1.81^{\circ}$ from a preoperative mean of
(99.18\%) were implanted within $90 \pm 3^{\circ}$ of the MPTA.

A significant correlation was observed between the femoral component position (mLDFA) and the postoperative HKA axis $(\mathrm{r}=-0.67, p<0.001$; 95\%CI: -0.73 to 0.595$)$ as well as between the tibial component position (MPTA) and the postoperative HKA axis $(\mathrm{r}=0.52$, $p<0.001$; $95 \%$ CI: 0.42 to 0.60$)$.

There was a close and significant $(p<0.0001)$ correlation between intra- and interobserver measurements for all primary variables (Table 4 ).

\section{Discussion}

The main findings of the present work are as follows: the HKA axis was within $180 \pm 3^{\circ}$ in $95.92 \%$ of knees, femoral components were implanted within $90 \pm 3^{\circ}$ in $97.14 \%$ of knees, and tibial components implanted 
Table 3 Comparison of outliers in primary variables in groups stratified by age, sex, side, BMI and type and severity of the deformity

\begin{tabular}{|c|c|c|c|}
\hline \multicolumn{4}{|l|}{ Radiographic variable } \\
\hline Age & $\leq 65$ years, $N=144$ & $>65$ years, $N=101$ & $p$ value \\
\hline HKA axis, Outlier (\%) & $06,(4.16 \%)$ & $04,(3.96 \%)$ & 1.00 \\
\hline mLDFA, Outlier (\%) & $05,(3.47 \%)$ & $02,(1.98 \%)$ & 0.70 \\
\hline MPTA, Outlier (\%) & $01,(0.69 \%)$ & 01, (0.99\%) & 1.00 \\
\hline Sex & Male, $N=52$ & Female, $N=193$ & \\
\hline HKA axis, Outlier (\%) & $01,(1.92 \%)$ & $09,(4.66 \%)$ & 0.69 \\
\hline mLDFA, Outlier (\%) & $00,(0.00 \%)$ & $07,(3.62 \%)$ & 0.35 \\
\hline MPTA, Outlier (\%) & $01,(1.92 \%)$ & $01,(0.51 \%)$ & 0.38 \\
\hline Side & Left, $N=116$ & Right, $N=129$ & \\
\hline HKA axis, Outlier (\%) & $05,(4.31 \%)$ & $05,(3.87 \%)$ & 1.00 \\
\hline mLDFA, Outlier (\%) & $03,(2.58 \%)$ & $01,(0.007 \%)$ & 0.34 \\
\hline MPTA, Outlier (\%) & $01,(0.86 \%)$ & $04,(3.10 \%)$ & 0.37 \\
\hline BMI & $\leq 30 \mathrm{~kg} / \mathrm{m}^{2}, N=124$ & $>\mathbf{3 0} \mathbf{k g} / \mathbf{m}^{2}, N=121$ & \\
\hline HKA axis, Outlier (\%) & $04,(3.22 \%)$ & $06,(4.95 \%)$ & 0.53 \\
\hline mLDFA, Outlier (\%) & $03,(2.41 \%)$ & $04,(3.30 \%)$ & 0.71 \\
\hline MPTA, Outlier (\%) & $01,(0.80 \%)$ & $01,(0.83 \%)$ & 1.00 \\
\hline Type severity of the deformity & Varus, $N=227$ & Valgus, $N=18$ & \\
\hline HKA axis, Outlier (\%) & $10,(4.40 \%)$ & $00,(0.00 \%)$ & 0.61 \\
\hline mLDFA, Outlier (\%) & $07,(3.08 \%)$ & $00,(0.00 \%)$ & 1.00 \\
\hline MPTA, Outlier (\%) & $02,(0.88 \%)$ & $00,(0.00 \%)$ & 1.00 \\
\hline Severity of the varus deformity & $\leq 15^{0}, N=193$ & $>15^{0}, N=34$ & \\
\hline HKA axis, Outlier (\%) & $07,(3.62 \%)$ & $03,(8.82 \%)$ & 0.17 \\
\hline mLDFA, Outlier (\%) & $06,(3.10 \%)$ & $01,(2.94 \%)$ & 1.00 \\
\hline MPTA, Outlier (\%) & $01,(0.51 \%)$ & $01,(2.94 \%)$ & 0.27 \\
\hline
\end{tabular}

Table 4 Intra-observer and inter-observer correlation for primary variables measured

\begin{tabular}{lll}
\hline Primary Variable Measured & $\begin{array}{l}\text { Intra-observer } \\
\text { Correlation }\end{array}$ & $\begin{array}{l}\text { Inter-observer } \\
\text { Correlation* }\end{array}$ \\
\hline Pre-operative HKA axis & 0.99 & 1.00 \\
Post-operative HKA axis & 0.99 & 0.98 \\
Pre-operative mLDFA & 0.98 & 0.98 \\
Post-operative mLDFA & 0.97 & 0.97 \\
Pre-operative MPTA & 0.99 & 0.99 \\
Post-operative MPTA & 0.96 & 0.95
\end{tabular}

$\$$ and $\#$ : $p$ value $<0.0001$ for all the variables measured

within $90 \pm 3^{\circ}$ in $99.18 \%$ of knees on the coronal plane. The rate of for HKA axis and femoral and tibial component position outliers in this series was $4.08 \%, 2.86 \%$, and $0.82 \%$, respectively. There were no outliers in the valgus deformity group. There were more outliers in the $>15^{\circ}$ varus deformity group than in the $<15^{\circ}$ varus deformity group The number of knees with valgus deformity and varus deformity $>15^{\circ}$ in the present study was small, so statistical significance could not be achieved for this finding. The reason for consistency in achieving the desired alignment by the described method could be that the majority of the potential errors leading to malalignment in TKA were bypassed by calculating the thickness of bone to be resected. Intraoperatively, the same thickness of bone was resected after removing cartilage and matching the thickness to that of the preoperative plan. The influence of age, sex, side, and BMI on the risk of postoperative limb malalignment and component malposition was also analyzed using the method described, and it was observed that none of these factors influenced any of the outcome variables, suggesting consistency of the method used (Table 3).

Published reports in the literature have described low rates of a neutral mechanical axis being achieved postoperatively using conventional jigs compared with CAS $[17,24,37]$ and robot-assisted TKA [36, 37], while the results of PSI are mixed [20,24,37]. This may be due to multiple reasons. On the femoral side, Bardakos et al. [5] found that in 30 to $51 \%$ of knees, the measured VCA was $>6^{\circ}$ or $<5^{\circ}$, and the VCA depended upon the neckshaft angle (NSA) and the horizontal offset of the hip. Mullaji et al. [27] also found that the VCA was variable, ranging from $3^{\circ}$ to $10^{\circ}$, and that it correlated with the severity of deformity and femoral bowing. Deakin 
et al. [12] compared the postoperative HKA axis in conventional jig-based TKA between two groups: group A, with a variable distal femoral VCA, and group $\mathrm{B}$, with a fixed VCA. They achieved an HKA axis within $180 \pm 3^{\circ}$ in $85 \%$ of knees in group A and $69 \%$ of knees in group B; however, they did not report individual component alignment. Interestingly, despite using a variable VCA, there were $15 \%$ outliers in group A, which may be due to the discrepancy between the plan and its execution. Lampart et al. [21] prospectively collected computed tomography (CT) data from 1480 consecutive patients who underwent $\mathrm{CT}$ for $3 \mathrm{D}$ reconstruction before TKA and observed a variable femoral mechanical (FMA) angle. They concluded that due to greater variability of the FMA angle, the FMA angle seems more relevant than the hip-kneeshaft (HKS) angle in defining the strategy of realignment of the lower limb. Palanisami et al. also observed in their study that a variable VCA leads to better alignment in TKA [29]. In the present series, a variable VCA (ranging from $3^{\circ}$ to $10^{\circ}$ ) based on the mLDFA for bone resection was used, and $13.06 \%$ of knees had a VCA different from the traditional $5-7^{\circ}$. Additionally, a eVCA different from the pVCA was observed in $8.16 \%$ of knees. mLDFAs within $90 \pm 3^{\circ}$ were achieved in $97.14 \%$ of knees, implying that the VCA is variable and that they further need adjustment intraoperatively to resect the planned thickness of bone. Although Kinzel et al. [19] implanted femoral components on the neutral femoral mechanical axis in $100 \%$ of 80 TKA procedures utilizing preoperative planning, they utilized CT scans for planning and postoperative evaluation. The accuracy of CT-based planning or the smaller sample size could be the reason for the better results of that study.

On the tibial side, in an attempt to improve the precision of proximal tibial resection, Magobotha et al. [25] used intraoperative fluoroscopy and achieved proximal tibial resection at $90 \pm 2^{\circ}$ on the coronal plane in $100 \%$ of a small cohort of 36 patients. Wu et al. [41] preoperatively planned proximal tibial resection on LLRs and achieved a neutral tibial implant position in $89.1 \%$ (114/128) of knees, while in the comparator group, in which they did not plan resection and depended on the extramedullary jig, they achieved a neutral tibial implant position in $79.1 \%$ of knees. The results of the current study support this trend, with a rate of tibial component position outliers of $0.82 \%(2 / 245)$. There were differences in the methodology of tibial resection between these two studies, which could be a reason for differences in the radiological outcomes. In the present study, a mark was aligned on the superior surface of the cutting block of the tibial jig to the line drawn from anterior to posterior along the center of the tibial spine ignoring the tibial tubercle. Wu et al. [40] measured the extent of resection from the anterior tibial margin before resection and without paring cartilage (the thickness of cartilage is variable), whereas in this study, it was measured from the center of the unaffected condyle and outer margin of the affected condyle after paring cartilage from the underlying bone before and after tibial resection to match the preoperative plan.

The reasons for the outliers observed in mechanical axis malalignment and component position in the present study were also considered. Potential reasons for these outliers include errors in the plan and its execution, differences in the center of the knee between preoperatively and postoperatively (lateral component placement causes overall varus and medial placement causes valgus), improper seating of components on one side, lifting of components during bone cement setting due to slight differences in medial and lateral ligament tension, and combinations of some or all of the above factors. Resolving these issues in future studies could potentially further reduce the number of outliers [18].

Limitations- The major limitation of this study is its single-arm design without any direct control arm for comparison with other methods, such as conventional surgery, CAS, PSI, or robotic-assisted surgery. Additionally, the number of knees in the $>15^{\circ}$ varus deformity group and the valgus deformity group was small. All surgeries were planned and executed by a single surgeon experienced in conventional knee replacement surgery, which might have resulted in planning and execution bias unknown to the authors. Hence, we suggest that a multisurgeon and multicentric randomized controlled trial be performed for the comparison of this technique with other techniques, such as CAS and robotic-assisted TKA, to test the reliability, repeatability, and validity of this technique. The strength of this study is its prospective design and fairly large sample size.

\section{Conclusion}

Given the favorable outcomes in terms of achieving desirable leg and component alignment in the present series, we conclude that TKA based on a modified method for preoperative planning (i.e., the true-alignment technique) described in this paper can produce reliable and consistent results without additional costs.

\section{Authors' contributions}

DS conceived of the presented idea and developed the theory, performed the surgical procedures, and wrote the manuscript with inputs from all the authors. KCP helped in the analysis. RDS helped in statistical analysis and editing of the manuscript. All authors discussed the results and provided critical feedback which helped shape the manuscript.

\section{Competing interests}

The authors declare that they have no competing interests. 


\section{Author details}

'Zydus Hospitals, Ahmedabad, Gujarat 380059, India. ${ }^{2}$ Department of Biochemistry, All India Institute of Medical Sciences, Khanderi, Rajkot, Gujarat, India.

Received: 22 June 2021 Accepted: 15 October 2021

Published online: 02 November 2021

\section{References}

1. Abdel MP, Oussedik S, Parratte S et al (2014) Coronal alignment in total knee replacement: historical review, contemporary analysis, and future direction. Bone Joint J 96-B:857-862

2. Akagi M, Oh M, Nonaka T et al (2004) An anteroposterior axis of the tibia for total knee arthroplasty. Clin Orthop Relat Res 420:213-219

3. Antonios JK, Korber S, Sivasundaram L et al (2019) Trends in computer navigation and robotic assistance for total knee arthroplasty in the United States: an analysis of patient and hospital factors. Arthroplast Today 5:88-95

4. Bae DK, Song SJ (2011) Computer assisted navigation in knee arthroplasty. Clin Orthop Surg 3:259-267

5. Bardakos N, Cil A, Thompson B et al (2007) Mechanical axis cannot be restored in total knee arthroplasty with a fixed valgus resection angle: a radiographic study. J Arthroplast 22(6 Suppl 2):85-89

6. Bardakos NV, Lilikakis AK (2014) Customised jigs in primary total knee replacement. Orthopedic Muscul Sys Curr Res S2:S2-007

7. Bäthis H, Perlick L, Tingart M et al (2005) Intraoperative cutting errors in total knee arthroplasty. Arch Orthop Trauma Surg 125:16-20

8. Buza JA, Vigdorchik J, Schwarzkopf R (2018) Robotics and the modern total knee arthroplasty. Tech Orthop 33:66-70

9. Chiu KY, Yau WP, Ng TP et al (2008) The accuracy of extramedullary guides for tibial component placement in total knee arthroplasty. Int Orthop 32:467-471

10. Choong PF, Dowsey MM, Stoney JD (2009) Does accurate anatomical alignment result in better function and quality of life? Comparing conventional and computer-assisted total knee arthroplasty. J Arthroplast 24:560-569

11. Cinotti G, Sessa P, D'Arino A et al (2015) Improving tibial component alignment in total knee arthroplasty. Knee Surg Sports Traumatol Arthrosc 23:3563-3570

12. Deakin AH, Sarungi M (2014) A comparison of variable angle versus fixed angle distal femoral resection in primary total knee arthroplasty. J Arthroplast 29:1133-1137

13. Dexel J, Kirschner S, Günther KP et al (2014) Agreement between radiological and computer navigation measurement of lower limb alignment. Knee Surg Sports Traumatol Arthrosc 22:2721-2727

14. Fang DM, Ritter MA, Davis KE (2009) Coronal alignment in total knee arthroplasty: just how important is it? J Arthroplast 24:39-43

15. Fukagawa S, Matsuda S, Mitsuyasu H et al (2011) Anterior border of the tibia as a landmark for extramedullary alignment guide in total knee arthroplasty for varus knees. J Orthop Res 29:919-924

16. Gøthesen O, Espehaug B, Havelin LI et al (2014) Functional outcome and alignment in computer-assisted and conventionally operated total knee replacements: a multicentre parallel-group randomised controlled trial. Bone Joint J 96-B(5):609-618

17. Hetaimish BM, Khan MM, Simunovic N et al (2012) Meta-analysis of navigation vs conventional total knee arthroplasty. J Arthroplast 27:1177-1182

18. Howie DF, Love GJ, Deakin AH et al (2015) Intra-operative deviation in limb alignment occurring at implantation in total knee arthroplasty. Knee 22:47-50

19. Kinzel V, Scaddan M, Bradley B et al (2004) Varus/valgus alignment of the femur in total knee arthroplasty. Can accuracy be improved by preoperative CT scanning? Knee 11:197-201

20. Kwon OR, Kang KT, Son J et al (2017) Patient-specific instrumentation development in TKA: 1st and 2nd generation designs in comparison with conventional instrumentation. Arch Orthop Trauma Surg 137:111-118
21. Lampart M, Behrend H, Moser LB et al (2019) Due to great variability fixed HKS angle for alignment of the distal cut leads to a significant error in coronal TKA orientation. Knee Surg Sports Traumatol Arthrosc 27:1434-1441

22. Liau JJ, Cheng CK, Huang CH et al (2002) The effect of malalignment on stresses in polyethylene component of total knee prostheses--a finite element analysis. Clin Biomech (Bristol, Avon) 17:140-146

23. Longstaff LM, Sloan K, Stamp N et al (2009) Good alignment after total knee arthroplasty leads to faster rehabilitation and better function. J Arthroplast 24:570-578

24. MacDessi SJ, Jang B, Harris IA et al (2014) A comparison of alignment using patient specific guides, computer navigation and conventional instrumentation in total knee arthroplasty. Knee 21:406-409

25. Magobotha SK, Mayet Z, Nyamuda RT (2018) An alternative method to improve the varus/valgus tibial alignment in total knee replacement. Orthopaedic Proceedings (Abstract) 96-B(19)

26. Mullaji A, Kanna R, Marawar S et al (2007) Comparison of limb and component alignment using computer-assisted navigation versus image intensifier-guided conventional total knee arthroplasty: a prospective, randomized, single-surgeon study of 467 knees. J Arthroplast 22:953-959

27. Mullaji AB, Shetty GM, Kanna R et al (2013) The influence of preoperative deformity on valgus correction angle: an analysis of 503 total knee arthroplasties. J Arthroplast 28:20-27

28. Novotny J, Gonzalez MH, Amirouche FM et al (2001) Geometric analysis of potential error in using femoral intramedullary guides in total knee arthroplasty. J Arthroplast 16:641-647

29. Palanisami D, lyyampillai $G$, Shanmugam S et al (2016) Individualised distal femoral cut improves femoral component placement and limb alignment during total knee replacement in knees with moderate and severe varus deformity. Int Orthop 40:2049-2054

30. Parratte S, Pagnano MW, Trousdale RT et al (2010) Effect of postoperative mechanical axis alignment on the fifteen-year survival of modern, cemented total knee replacements. J Bone Joint Surg Am 15:2143-2149

31. Picard F, Clarke J, Deep K et al (2014) Computer assisted knee replacement surgery: is the movement mainstream? Orthopedic Muscul Syst 3:153

32. Reed SC, Gollish J (1997) The accuracy of femoral intramedullary guides in total knee arthroplasty. J Arthroplast 12:677-682

33. Rivière C, Iranpour F, Auvinet E et al (2017) Alignment options for total knee arthroplasty: a systematic review. Orthop Traumatol Surg Res 103:1047-1056

34. Shetty GM, Mullaji A, Bhayde S et al (2014) Factors contributing to inherent varus alignment of lower limb in normal Asian adults: role of tibial plateau inclination. Knee 21:544-548

35. Sloan M, Premkumar A, Sheth NP (2018) Projected volume of primary Total joint Arthroplasty in the U.S., 2014 to 2030. J Bone Joint Surg Am 100:1455-1460

36. Song EK, Seon JK, Park SJ et al (2011) Simultaneous bilateral total knee arthroplasty with robotic and conventional techniques: a prospective, randomized study. Knee Surg Sports Traumatol Arthrosc 19:1069-1076

37. Thienpont E, Fennema P, Price A (2013) Can technology improve alignment during knee arthroplasty. Knee 20(Suppl 1):S21-S28

38. Thienpont E, Parvizi J (2016) A new classification for the Varus knee. J Arthroplast 31:2156-2160

39. Werner FW, Ayers DC, Maletsky LP et al (2005) The effect of valgus/varus malalignment on load distribution in total knee replacements. J Biomech 38:349-355

40. Williams D, Metcalfe A, Madete J et al (2020) The relationship between alignment, function and loading in total knee replacement: in-vivo analysis of a unique patient population. J Biomech 112:110042

41. Wu PH, Zhang ZQ, Fang SY et al (2016) Preoperative measurement of Tibial resection in Total knee Arthroplasty improves accuracy of postoperative limb alignment restoration. Chin Med J 129:2524-2529

\section{Publisher's Note}

Springer Nature remains neutral with regard to jurisdictional claims in published maps and institutional affiliations. 